\title{
SUPPLEMENTARY INFORMATION: FACILE DETERMINATION OF PHOSPHORYLATION SITES IN PEPTIDES USING TWO-DIMENSIONAL MASS SPECTROMETRY
}

Johanna Paris ${ }^{\dagger}$, Tomos E. Morgan ${ }^{\dagger}$, Christopher A. Wootton ${ }^{\dagger}$, Mark P. Barrow ${ }^{\dagger}$, John O’Hara Peter B. O’Connor*†

†University of Warwick, Department of Chemistry, Coventry, CV4 7AL, United Kingdom

*UCB, 216 Bath Road, Slough, SL1 3WE, United Kingdom

*Corresponding author email address: p.oconnor@,warwick.ac.uk

\section{TABLE OF CONTENTS}

Figures .2

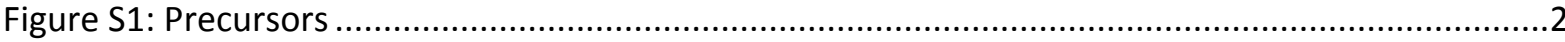

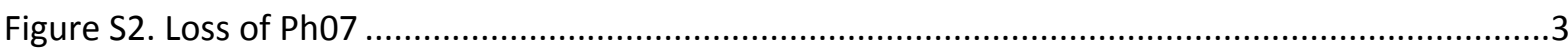

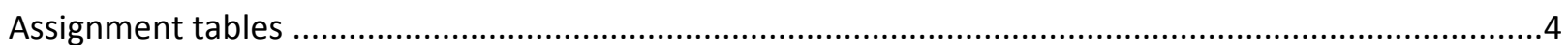

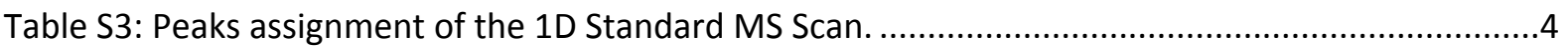

Table S4: Peaks assignment of the extracted autocorrelation line of the 2DMS spectrum. .................4

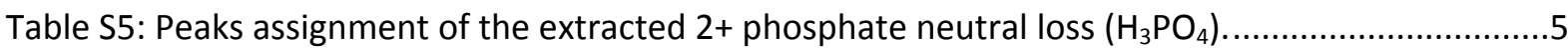

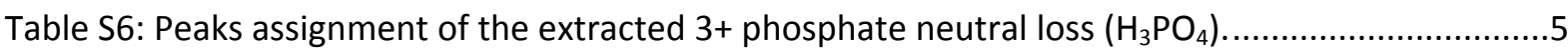

Table S7: Peaks assignment of the extracted $[\mathrm{Ph01}+2 \mathrm{H}]^{2+}$ precursor line. .........................................5

Table S8: Peaks assignment of the extracted $[\mathrm{Ph02}+2 \mathrm{H}]^{2+}$ precursor line. ........................................5

Table S9: Peaks assignment of the extracted $[\mathrm{Ph03}+2 \mathrm{H}]^{2+}$ precursor line. .......................................6

Table S10: Peaks assignment of the extracted $[\mathrm{Ph04}+2 \mathrm{H}]^{2+}$ precursor line. .......................................6

Table S11: Peaks assignment of the extracted $[\mathrm{Ph05}+2 \mathrm{H}]^{2+}$ precursor line. .........................................

Table S12: Peaks assignment of the extracted $[\mathrm{Ph08}+2 \mathrm{H}]^{2+}$ precursor line. .......................................

Table S13: Peaks assignment of the extracted $[\mathrm{Ph06}+2 \mathrm{H}]^{2+}$ precursor line. ......................................

Table S14: Peaks assignment of the extracted $[\mathrm{Ph09}+3 \mathrm{H}]^{3+}$ precursor line. .................................... 8

Table S15: Peaks assignment of the extracted $[\mathrm{Ph} 10+3 \mathrm{H}]^{3+}$ precursor line. ....................................... 


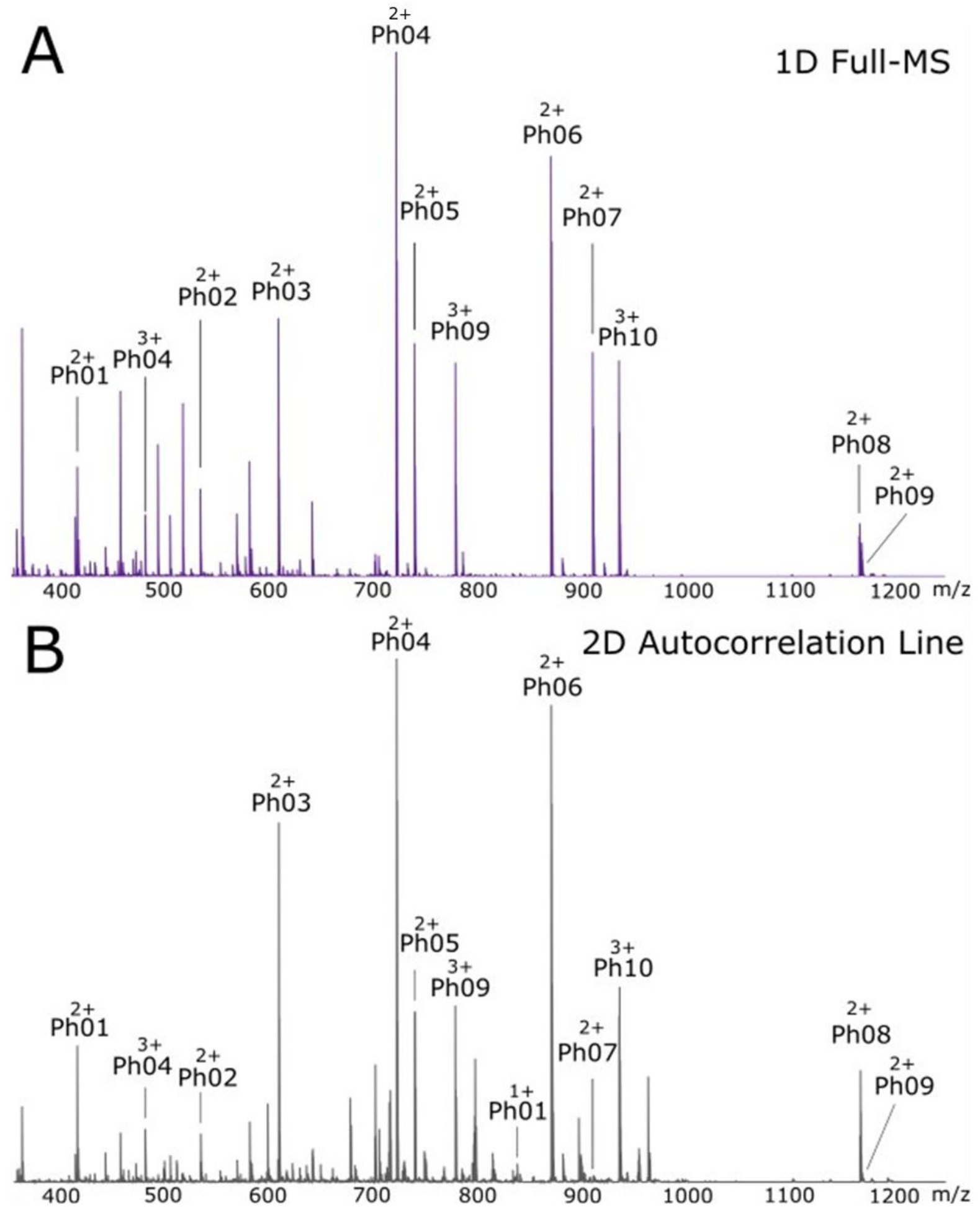

Figure S1: Precursors A: 1D standard MS scan B: Extracted 2D autocorrelation line 


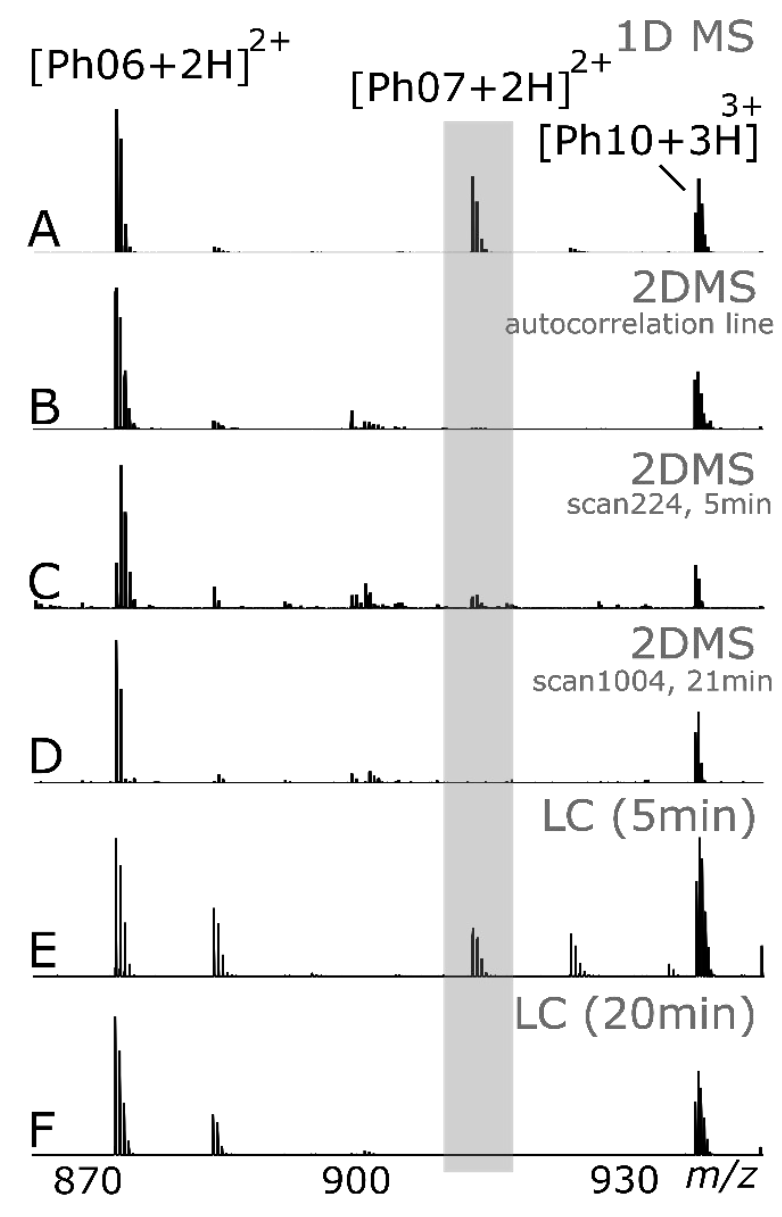

Figure S2. Loss of Ph07 A: 1D standard MS scan B: Extracted 2D autocorrelation line C: Extracted 1D scan of the 2DMS experiment (scan \#224 at $4.75 \mathrm{~min}$ ) D: Extracted 1D scan of the 2DMS experiment (scan \#1004 at 21min). E: Extracted 1D scan of the LCMS experiment (at $5 \mathrm{~min}$ ). F: Extracted 1D scan of the LCMS experiment (at 20min). 


\section{Assignment tables}

Absolute error is calculated as the average of the absolute ppm errors of all the assigned peaks. Absolute standard deviation is calculated as the standard deviation of the absolute ppm errors of all the assigned peaks.

\begin{tabular}{|c|c|c|c|}
\hline Species & Theoretical $\mathrm{m} / \mathrm{z}$ & Observed $\mathrm{m} / \mathrm{z}$ & $\mathrm{ppm}$ \\
\hline$[\mathrm{Ph01+2 \textrm {H }}]^{2+}$ & 418.19473 & 418.19493 & 0.467 \\
\hline$[\mathrm{Ph02+2H}]^{2+}$ & $\mathbf{5 3 6 . 2 1 0 2 6}$ & $\mathbf{5 3 6 . 2 1 0 2 6}$ & $\mathbf{- 0 . 0 0 6}$ \\
\hline$[\mathrm{PhO3+2H}]^{2+}$ & 611.26662 & 611.26651 & -0.175 \\
\hline$[\mathrm{Ph04+2H}]^{2+}$ & $\mathbf{7 2 3 . 8 5 9 5 0}$ & $\mathbf{7 2 3 . 8 5 9 5 1}$ & 0.014 \\
\hline$[\mathrm{Ph05+2H}]^{2+}$ & $\mathbf{7 4 1 . 3 1 7 0 3}$ & $\mathbf{7 4 1 . 3 1 7 0 3}$ & $\mathbf{- 0 . 0 0 7}$ \\
\hline$[\mathrm{Ph06+2H}]^{2+}$ & 872.34585 & 872.34632 & 0.537 \\
\hline$[\mathrm{Ph07+2H}]^{2+}$ & 912.32902 & 912.32926 & 0.266 \\
\hline$[\mathrm{Ph08+2H}]^{2+}$ & $\mathbf{1 1 6 7 . 9 2 5 9 2}$ & $\mathbf{1 1 6 7 . 9 2 5 9 4}$ & $\mathbf{0 . 0 1 5}$ \\
\hline$[\mathrm{Ph09+2H}]^{2+}$ & 1170.00888 & 1170.00892 & 0.034 \\
\hline$[\mathrm{Ph09+3H}]^{3+}$ & $\mathbf{7 8 0 . 3 4 1 6 8}$ & $\mathbf{7 8 0 . 3 4 1 5 6}$ & -0.152 \\
\hline$[\mathrm{Ph10+3H}]^{3+}$ & $\mathbf{9 3 7 . 4 0 5 2 3}$ & $\mathbf{9 3 7 . 4 0 5 2 1}$ & $\mathbf{- 0 . 0 2 2}$ \\
\hline
\end{tabular}

Table S3: Peaks assignment of the 1D Standard MS Scan. Internal calibration points are in bold. Absolute error is $0.154 \mathrm{ppm}$, absolute standard deviation is $0.184 \mathrm{ppm}$.

\begin{tabular}{|c|c|c|c|}
\hline Species & Theoretical $\mathrm{m} / \mathrm{z}$ & Observed $\mathrm{m} / \mathrm{z}$ & $\mathrm{ppm}$ \\
\hline$[\mathrm{Ph01+H}]^{1+}$ & 835.38219 & 835.38205 & -0.172 \\
\hline$[\mathrm{Ph01+2H}]^{2+}$ & $\mathbf{4 1 8 . 1 9 4 7 3}$ & $\mathbf{4 1 8 . 1 9 4 7 3}$ & $\mathbf{- 0 . 0 1 2}$ \\
\hline$[\mathrm{Ph02+2H}]^{2+}$ & 536.21026 & 536.21168 & 2.642 \\
\hline$[\mathrm{Ph03+2H}]^{2+}$ & $\mathbf{6 1 1 . 2 6 6 6 2}$ & $\mathbf{6 1 1 . 2 6 6 6 3}$ & $\mathbf{0 . 0 2 2}$ \\
\hline$[\mathrm{Ph03+3H}]^{3+}$ & 407.84684 & 407.84663 & -0.507 \\
\hline$[\mathrm{Ph04+2H}]^{2+}$ & 723.85950 & 723.85961 & 0.152 \\
\hline$[\mathrm{Ph04+3H}]^{3+}$ & 482.90876 & 482.90879 & 0.065 \\
\hline$[\mathrm{Ph05+2H}]^{2+}$ & 741.31703 & 741.31739 & 0.479 \\
\hline$[\mathrm{Ph06+2H}]^{2+}$ & 872.34585 & 872.34567 & -0.208 \\
\hline$[\mathrm{Ph07+2H}]^{2+}$ & 912.32902 & 912.32889 & -0.140 \\
\hline$[\mathrm{Ph08+2H}]^{2+}$ & 1167.92592 & 1167.92205 & -3.316 \\
\hline$[\mathrm{Ph09+2H}]^{2+}$ & 1170.00888 & 1170.00827 & -0.522 \\
\hline$[\mathrm{Ph09+3H}]^{3+}$ & $\mathbf{7 8 0 . 3 4 1 6 8}$ & $\mathbf{7 8 0 . 3 4 1 6 4}$ & $\mathbf{- 0 . 0 5 0}$ \\
\hline$[\mathrm{Ph10+3H}]^{3+}$ & $\mathbf{9 3 7 . 4 0 5 2 3}$ & $\mathbf{9 3 7 . 4 0 5 2 6}$ & $\mathbf{0 . 0 3 2}$ \\
\hline
\end{tabular}

Table S4: Peaks assignment of the extracted autocorrelation line of the 2DMS spectrum. Internal calibration points are in bold. Absolute error is $0.594 \mathrm{ppm}$, absolute standard deviation is $0.997 \mathrm{ppm}$. 


\begin{tabular}{|c|c|c|c|}
\hline Species & Theoretical $\mathrm{m} / \mathrm{z}$ & Observed $\mathrm{m} / \mathrm{z}$ & ppm \\
\hline$\left[\mathrm{Ph01}+2 \mathrm{H}-\mathrm{H}_{3} \mathrm{PO}_{4}\right]^{2+}$ & 369.20629 & 369.20629 & 0.008 \\
\hline$\left[\mathrm{PhO2}+2 \mathrm{H}-\mathrm{H}_{3} \mathrm{PO}_{4}\right]^{2+}$ & 487.22182 & 487.22221 & 0.810 \\
\hline$\left[\mathrm{PhO3}+2 \mathrm{H}-\mathrm{H}_{3} \mathrm{PO}_{4}\right]^{2+}$ & 562.27817 & 562.27793 & -0.425 \\
\hline$\left[\mathrm{PhO} 4+2 \mathrm{H}-\mathrm{H}_{3} \mathrm{PO}_{4}\right]^{2+}$ & 674.87105 & 674.87065 & -0.596 \\
\hline$\left[\mathrm{Ph05}+2 \mathrm{H}-\mathrm{H}_{3} \mathrm{PO}_{4}\right]^{2+}$ & 692.32859 & 692.32854 & -0.068 \\
\hline$\left[\mathrm{Ph06}+2 \mathrm{H}-\mathrm{H}_{3} \mathrm{PO}_{4}\right]^{2+}$ & 823.35740 & 823.35744 & 0.044 \\
\hline$\left[\mathrm{Ph08}+2 \mathrm{H}-\mathrm{H}_{3} \mathrm{PO}_{4}\right]^{2+}$ & 1118.93748 & 1118.93751 & 0.031 \\
\hline
\end{tabular}

Table S5: Peaks assignment of the extracted 2+ phosphate neutral loss $\left(\mathrm{H}_{3} \mathrm{PO}_{4}\right)$. Internal calibration points are in bold. Absolute error is $0.283 \mathrm{ppm}$; absolute standard deviation is $0.301 \mathrm{ppm}$.

\begin{tabular}{|c|c|c|c|}
\hline Species & Theoretical $\mathrm{m} / \mathrm{z}$ & Observed $\mathrm{m} / \mathrm{z}$ & $\mathrm{ppm}$ \\
\hline$\left[\mathrm{PhO2+3H}-\mathrm{H}_{3} \mathrm{PO}_{4}\right]^{3+}$ & 325.15030 & 325.15030 & -0.007 \\
\hline$\left[\mathrm{PhO} 0+3 \mathrm{H}-\mathrm{H}_{3} \mathrm{PO}_{4}\right]^{3+}$ & 450.24979 & 450.24980 & 0.017 \\
\hline 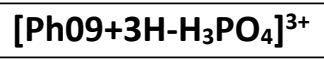 & 747.68271 & 747.68267 & -0.059 \\
\hline$\left[\mathrm{Ph} 10+3 \mathrm{H}-\mathrm{H}_{3} \mathrm{PO}_{4}\right]^{3+}$ & 904.74627 & 904.74631 & 0.050 \\
\hline
\end{tabular}

Table S6: Peaks assignment of the extracted 3+ phosphate neutral loss $\left(\mathrm{H}_{3} \mathrm{PO}_{4}\right)$. Internal calibration points are in bold. Absolute error is $0.033 \mathrm{ppm}$; absolute standard deviation is $0.021 \mathrm{ppm}$.

\begin{tabular}{|c|c|c|c|}
\hline Species & Theoretical $\mathrm{m} / \mathrm{z}$ & Observed $\mathrm{m} / \mathrm{z}$ & ppm \\
\hline$[\mathrm{PhO1+2 \textrm {H }}]^{2+}$ & 418.19473 & 418.19462 & -0.275 \\
\hline$\left[\mathrm{PhO} 01+2 \mathrm{H}-\mathrm{H}_{2} \mathrm{O}\right]^{2+}$ & 409.18945 & 409.18938 & -0.177 \\
\hline$\left[\mathrm{PhO1}+2 \mathrm{H}-\mathrm{H}_{3} \mathrm{PO}_{4}\right]^{2+}$ & 369.20629 & 369.20634 & 0.143 \\
\hline b2 & 213.15975 & 213.15975 & -0.020 \\
\hline b3 & 350.21867 & 350.21873 & 0.182 \\
\hline $\mathrm{b} 4$ & 437.25069 & 437.25111 & 0.950 \\
\hline py5 & 623.22972 & 623.22979 & 0.120 \\
\hline
\end{tabular}

Table S7: Peaks assignment of the extracted $[\mathbf{P h 0 1 + 2 H}]^{2+}$ precursor line. $\mathrm{Ph} 01$ : VLHSGpSR. Internal calibration points are in bold. Absolute error is $0.267 \mathrm{ppm}$; absolute standard deviation is $0.288 \mathrm{ppm}$.

\begin{tabular}{|c|c|c|c|}
\hline Species & Theoretical $\mathrm{m} / \mathrm{z}$ & Observed $\mathrm{m} / \mathrm{z}$ & $\mathrm{ppm}$ \\
\hline$[\mathrm{PhO2}+2 \mathrm{H}]^{2+}$ & 536.21026 & 536.21035 & 0.162 \\
\hline$\left[\mathrm{PhO} 2+2 \mathrm{H}-\mathrm{H}_{2} \mathrm{O}\right]^{2+}$ & 527.20498 & 527.20508 & 0.188 \\
\hline$\left[\mathrm{PhO} 2+2 \mathrm{H}-\mathrm{H}_{3} \mathrm{PO}_{4}\right]^{2+}$ & 487.22182 & 487.22192 & 0.215 \\
\hline b3 & 407.20374 & 407.20373 & -0.035 \\
\hline pb5 & 730.30321 & 730.30298 & -0.321 \\
\hline pb6 & 817.33524 & 817.33544 & 0.241 \\
\hline
\end{tabular}

Table S8: Peaks assignment of the extracted $[\mathbf{P h 0 2}+\mathbf{2 H}]^{2+}$ precursor line. Ph02: RSpYpSRSR. Internal calibration points are in bold. Absolute error is $0.194 \mathrm{ppm}$; absolute standard deviation is $0.087 \mathrm{ppm}$. 


\begin{tabular}{|c|c|c|c|}
\hline Species & Theoretical $\mathrm{m} / \mathrm{z}$ & Observed $\mathrm{m} / \mathrm{z}$ & ppm \\
\hline$[\mathrm{PhO3}+2 \mathrm{H}]^{2+}$ & 611.26662 & 611.26653 & -0.142 \\
\hline$\left[\mathrm{PhO3}+2 \mathrm{H}-\mathrm{H}_{2} \mathrm{O}\right]^{2+}$ & 602.26133 & 602.26167 & 0.557 \\
\hline$\left[\mathrm{PhO3}+2 \mathrm{H}-\mathrm{H}_{3} \mathrm{PO}_{4}\right]^{2+}$ & 562.27817 & 562.27845 & 0.500 \\
\hline b2 & 272.13533 & 272.13525 & -0.296 \\
\hline b3 & 359.16736 & 359.16694 & -1.166 \\
\hline b4 & 472.25142 & 472.25146 & 0.079 \\
\hline pb8 & 960.38225 & 960.38566 & 3.548 \\
\hline$y 2$ & 262.15098 & 262.15098 & -0.002 \\
\hline y3 & 349.18301 & 349.18313 & 0.347 \\
\hline y4 & 512.24634 & 512.24665 & 0.610 \\
\hline py6 & 750.28181 & 750.28237 & 0.746 \\
\hline py7 & 863.36587 & 863.36522 & -0.758 \\
\hline py8 & 950.39790 & 950.39797 & 0.070 \\
\hline
\end{tabular}

Table S9: Peaks assignment of the extracted $[\mathbf{P h 0 3}+\mathbf{2 H}]^{2+}$ precursor line. $\mathrm{Ph} 03$ : RDSLGpTYSSR. Internal calibration points are in bold. Absolute error is $0.678 \mathrm{ppm}$; absolute standard deviation is $0.889 \mathrm{ppm}$.

\begin{tabular}{|c|c|c|c|}
\hline Species & Theoretical $\mathrm{m} / \mathrm{z}$ & Observed $\mathrm{m} / \mathrm{z}$ & ppm \\
\hline$\left[\mathrm{PhO} 4+2 \mathrm{H}-\mathrm{H}_{3} \mathrm{PO}_{4}-\mathrm{HPO}_{3}\right]^{2+}$ & 634.88789 & 634.88792 & 0.053 \\
\hline$\left[\mathrm{PhO} 4+2 \mathrm{H}-2 \mathrm{H}_{3} \mathrm{PO}_{4}\right]^{2+}$ & 625.88260 & 625.88262 & 0.025 \\
\hline$\left[\mathrm{PhO} 4+2 \mathrm{H}-\mathrm{H}_{3} \mathrm{PO}_{4}\right]^{2+}$ & 674.87105 & 674.87096 & -0.136 \\
\hline 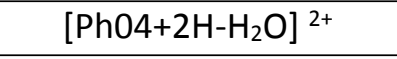 & 714.85422 & 714.85416 & -0.080 \\
\hline$[\mathrm{Ph04+2H}]^{2+}$ & 723.85950 & 723.85954 & 0.056 \\
\hline b4 & 456.31805 & 456.31832 & 0.601 \\
\hline ppb7 & 958.44103 & 958.44203 & 1.046 \\
\hline ppb8 & 1114.54214 & 1114.54497 & 2.540 \\
\hline ppb9 & 1229.56908 & 1229.56903 & -0.042 \\
\hline ppb10 & 1300.60620 & 1300.60556 & -0.489 \\
\hline y2 & 218.14992 & 218.14992 & 0.009 \\
\hline y3 & 333.17686 & 333.17681 & -0.153 \\
\hline y4 & 489.27797 & 489.27796 & -0.024 \\
\hline y5 & 602.36204 & 602.36204 & 0.007 \\
\hline py7 & 911.43462 & 911.43290 & -1.890 \\
\hline py9 & 1137.60275 & 1137.60253 & -0.194 \\
\hline
\end{tabular}

Table S10: Peaks assignment of the extracted $[\mathbf{P h 0 4}+\mathbf{2 H}]^{2+}$ precursor line. Ph04: pTKLIpTQLRDAK. Internal calibration points are in bold. Absolute error is $0.459 \mathrm{ppm}$; absolute standard deviation is $0.726 \mathrm{ppm}$. 


\begin{tabular}{|c|c|c|c|}
\hline Species & Theoretical $m / z$ & Observed $m / z$ & ppm \\
\hline$[\mathrm{PhO5}+2 \mathrm{H}]^{2+}$ & 741.31703 & 741.31680 & -0.317 \\
\hline$\left[\mathrm{Ph05}+2 \mathrm{H}-\mathrm{H}_{2}\right]^{2+}$ & 732.31175 & 732.31162 & -0.181 \\
\hline$\left[\mathrm{Ph05}+2 \mathrm{H}-\mathrm{H}_{3} \mathrm{PO}_{4}\right]^{2+}$ & 692.32859 & 692.32841 & -0.256 \\
\hline b3 & 357.17686 & 357.17681 & -0.143 \\
\hline b4 & 428.21397 & 428.21435 & 0.876 \\
\hline b5 & 557.25657 & 557.25670 & 0.237 \\
\hline b6 & 685.31515 & 685.31619 & 1.524 \\
\hline b9 & 956.43197 & 956.43092 & -1.094 \\
\hline y2 & $\mathbf{2 7 2 . 1 7 1 7 2}$ & $\mathbf{2 7 2 . 1 7 1 7 2}$ & $\mathbf{0 . 0 1 5}$ \\
\hline y3 & 359.20374 & 359.20373 & -0.040 \\
\hline py4 & $\mathbf{5 2 6 . 2 0 2 1 0}$ & $\mathbf{5 2 6 . 2 0 2 1 6}$ & $\mathbf{0 . 1 0 7}$ \\
\hline py5 & 613.23413 & 613.23302 & -1.813 \\
\hline py6 & $\mathbf{7 0 0 . 2 6 6 1 6}$ & $\mathbf{7 0 0 . 2 6 6 0 4}$ & $\mathbf{- 0 . 1 7 2}$ \\
\hline py7 & 797.31892 & $\mathbf{7 9 7 . 3 1 9 3 9}$ & 0.584 \\
\hline py10 & $\mathbf{1 1 2 5 . 4 5 7 2 1}$ & $\mathbf{1 1 2 5 . 4 5 7 3 2}$ & $\mathbf{0 . 0 9 9}$ \\
\hline
\end{tabular}

Table S11: Peaks assignment of the extracted $[\mathbf{P h 0 5}+\mathbf{2 H}]^{2+}$ precursor line. Ph05: EVQAEQPSSpSSPR. Internal calibration points are in bold. Absolute error is $0.497 \mathrm{ppm}$; absolute standard deviation is $0.550 \mathrm{ppm}$.

\begin{tabular}{|c|c|c|c|}
\hline Species & Theoretical $\mathrm{m} / \mathrm{z}$ & Observed $\mathrm{m} / \mathrm{z}$ & $\mathrm{ppm}$ \\
\hline$[\mathrm{PhO} 8+2 \mathrm{H}]^{2+}$ & 1167.92592 & 1167.92646 & 0.460 \\
\hline$\left[\mathrm{PhO} 8+2 \mathrm{H}-\mathrm{H}_{2} \mathrm{O}\right]^{2+}$ & 1158.92064 & 1158.92141 & 0.664 \\
\hline$\left[\mathrm{Ph08}+2 \mathrm{H}-\mathrm{H}_{3} \mathrm{PO}_{4}\right]^{2+}$ & 1118.93748 & 1118.93760 & 0.112 \\
\hline b3 & 392.14523 & 392.14502 & -0.527 \\
\hline b4 & 521.18782 & 521.18844 & 1.190 \\
\hline b8 & 853.33627 & 853.33639 & 0.135 \\
\hline pb16 & 1767.59053 & 1767.59037 & -0.089 \\
\hline y2 & 276.15540 & 276.15540 & 0.010 \\
\hline y3 & 405.19799 & 405.19791 & -0.198 \\
\hline y4 & 568.26132 & 568.26123 & -0.156 \\
\hline y5 & 683.28826 & 683.28658 & -2.461 \\
\hline y6 & 740.30973 & 740.30970 & -0.035 \\
\hline y7 & 841.35740 & 841.35701 & -0.468 \\
\hline py10 & 1224.43039 & 1224.43227 & 1.539 \\
\hline py14 & 1686.60545 & 1686.60456 & -0.527 \\
\hline py16 & 1814.66403 & 1814.66177 & -1.243 \\
\hline py172+ & 972.35695 & 972.35730 & 0.362 \\
\hline py18 ${ }^{2+}$ & 1029.87042 & 1029.87137 & 0.923 \\
\hline
\end{tabular}

Table S12: Peaks assignment of the extracted $[\mathbf{P h 0 8}+\mathbf{2 H}]^{2+}$ precursor line. Ph08: FEDEGAGFEESpSETGDYEEK. Internal calibration points are in bold. Absolute error is $0.617 \mathrm{ppm}$; absolute standard deviation is $0.626 \mathrm{ppm}$. 


\begin{tabular}{|c|c|c|c|}
\hline Species & Theoretical $\mathrm{m} / \mathrm{z}$ & Observed $\mathrm{m} / \mathrm{z}$ & $\mathrm{ppm}$ \\
\hline$[\mathrm{PhO6}+2 \mathrm{H}]^{2+}$ & 872.34585 & 872.34577 & -0.094 \\
\hline$\left[\mathrm{PhO6}+2 \mathrm{H}-\mathrm{H}_{2} \mathrm{O}\right]^{2+}$ & 863.34057 & 863.34079 & 0.255 \\
\hline$\left[\mathrm{Ph06}+2 \mathrm{H}-\mathrm{H}_{3} \mathrm{PO}_{4}\right]^{2+}$ & 823.35740 & 823.35756 & 0.189 \\
\hline b2 & 187.07133 & 187.07133 & -0.018 \\
\hline b3 & 316.11393 & 316.11398 & 0.170 \\
\hline pb6 & 667.19708 & 667.19731 & 0.348 \\
\hline pb7 & 796.23967 & 796.24088 & 1.518 \\
\hline $\mathrm{pb} 8$ & 925.28226 & 925.28132 & -1.020 \\
\hline pb10 & 1127.34124 & 1127.34253 & 1.148 \\
\hline pb11 & 1240.42530 & 1240.42449 & -0.652 \\
\hline pb12 & 1369.46789 & 1369.46866 & 0.560 \\
\hline pb14 & 1597.57890 & 1597.57916 & 0.163 \\
\hline $\mathrm{y} 2$ & 262.13975 & 262.13974 & -0.027 \\
\hline y3 & 375.22381 & 375.22341 & -1.069 \\
\hline y4 & 504.26640 & 504.26576 & -1.277 \\
\hline$y 4^{\prime}$ & 486.25584 & 486.25586 & 0.042 \\
\hline y5 & 617.35047 & 617.35043 & -0.062 \\
\hline y6 & 732.37741 & 732.37771 & 0.408 \\
\hline y7 & 819.40944 & 819.40935 & -0.109 \\
\hline$y 7^{\prime}$ & 801.39888 & 801.39894 & 0.081 \\
\hline y8 & 948.45203 & 948.45166 & -0.393 \\
\hline$y 8^{\prime}$ & 930.44147 & 930.44109 & -0.406 \\
\hline y9 & 1077.49463 & 1077.49366 & -0.897 \\
\hline y9' & 1059.48406 & 1059.48406 & -0.001 \\
\hline y10 & 1164.52665 & 1164.52659 & -0.055 \\
\hline py11 & 1331.52501 & 1331.52603 & 0.763 \\
\hline py12 & 1428.57778 & 1428.57614 & -1.146 \\
\hline py132+ & 779.31382 & 779.31334 & -0.620 \\
\hline
\end{tabular}

Table S13: Peaks assignment of the extracted $[\mathbf{P h 0 6}+\mathbf{2 H}]^{2+}$ precursor line. Ph06: ADEPpSSEESDLEIDK. Internal calibration points are in bold. ' $:$ water loss. Absolute error is $0.482 \mathrm{ppm}$; absolute standard deviation is $0.446 \mathrm{ppm}$.

\begin{tabular}{|c|c|c|c|}
\hline Species & Theoretical $\mathrm{m} / \mathrm{z}$ & Observed $\mathrm{m} / \mathrm{z}$ & $\mathrm{ppm}$ \\
\hline$[\mathrm{Ph} 09+3 \mathrm{H}]^{3+}$ & 780.34168 & 780.34143 & -0.319 \\
\hline$\left[\mathrm{Ph09}+3 \mathrm{H}-\mathrm{H}_{2} \mathrm{O}\right]^{3+}$ & 774.33816 & 774.33767 & -0.629 \\
\hline 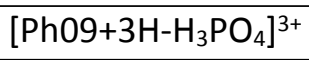 & 747.68271 & 747.68227 & -0.593 \\
\hline $\mathrm{ppb} 17^{2+}$ & 1009.41884 & 1009.42166 & 2.798 \\
\hline y3 & 451.22996 & 451.22996 & 0.002 \\
\hline y5 & 661.36679 & 661.36688 & 0.141 \\
\hline py7 & 885.38661 & 885.38678 & 0.192 \\
\hline
\end{tabular}

Table S14: Peaks assignment of the extracted $[\mathbf{P h 0 9 + 3 H}]^{3+}$ precursor line. Ph09: ELSNpSPLRENSFGpSPLEFR. Internal calibration points are in bold. Absolute error is $0.668 \mathrm{ppm}$; absolute standard deviation is $0.896 \mathrm{ppm}$. 


\begin{tabular}{|c|c|c|c|}
\hline Species & Theoretical $\mathrm{m} / \mathrm{z}$ & Observed $\mathrm{m} / \mathrm{z}$ & $\mathrm{ppm}$ \\
\hline$[\mathrm{Ph10+3H}]^{3+}$ & $\mathbf{9 3 7 . 4 0 5 2 3}$ & $\mathbf{9 3 7 . 4 0 5 7 7}$ & $\mathbf{0 . 5 7 6}$ \\
\hline$\left[\mathrm{Ph} 10+3 \mathrm{H}-\mathrm{H}_{2} \mathrm{O}^{3+}\right.$ & 931.40171 & 931.40246 & 0.807 \\
\hline$\left[\mathrm{Ph} 10+3 \mathrm{H}-\mathrm{H}_{3} \mathrm{PO}_{4}\right]^{3+}$ & 904.74627 & 904.74693 & 0.735 \\
\hline b7 & 844.34717 & 844.34840 & 1.452 \\
\hline $\mathrm{pb} 16-2+$ & 1016.43547 & 1016.43099 & -4.410 \\
\hline pb18-2+ & 1115.48569 & 1115.48939 & 3.313 \\
\hline y3 & $\mathbf{4 0 0 . 2 3 0 2 9}$ & $\mathbf{4 0 0 . 2 3 0 2 9}$ & $-\mathbf{0 . 0 0 9}$ \\
\hline py6 & $\mathbf{7 7 9 . 3 4 4 7 5}$ & 779.34509 & 0.442 \\
\hline py7 & $\mathbf{9 3 5 . 4 4 5 8 6}$ & $\mathbf{9 3 5 . 4 4 5 7 5}$ & $\mathbf{- 0 . 1 1 4}$ \\
\hline ppy15 & $\mathbf{9 8 3 . 9 3 4 2 6}$ & $\mathbf{9 8 3 . 9 3 3 2 3}$ & $\mathbf{- 1 . 0 4 5}$ \\
\hline
\end{tabular}

Table S15: Peaks assignment of the extracted $[\mathbf{P h 1 0}+\mathbf{3 H}]^{3+}$ precursor line. Ph10: SPTEYHEPVpYANPFYRPTpTPQR. Internal calibration points are in bold. Absolute error is $1.290 \mathrm{ppm}$; absolute standard deviation is $1.368 \mathrm{ppm}$. 